\title{
Derivation of Pareto Optimal Operating Policies Based on Safety Indices for a Catalytic Multi-tubular Reactor Used for Nitrobenzene Hydrogenation
}

\author{
G. Maria, ${ }^{*}$ H. H. S. Khwayyir, ${ }^{\S}$ and D. Dinculescu \\ Department of Chemical and Biochemical Engineering, \\ University POLITEHNICA of Bucharest
}

doi: 10.15255/CABEQ.2015.2299

Original scientific paper Received: September 15, 2015 Accepted: September 3, 2016

\begin{abstract}
When highly exothermic/hazardous reactions are conducted in the presence of parametric uncertainty, derivation of optimal operating policies for a chemical reactor has to simultaneously consider several objectives of sustainability. The paper uses an original methodology to generate the Pareto optimal solutions when reactor productivity and safety objectives (expressed in probabilistic terms) are simultaneously considered using the process and reactor model in a simple way, in the presence of technological constraints, uncertainty in safety boundaries, and random fluctuations in control variables. An example is provided for the industrial fixed-bed tubular reactor used for the catalytic hydrogenation of nitrobenzene to aniline in vapour-phase.
\end{abstract}

Key words:

chemical reactor failure probability, runaway boundaries, Pareto optimal policies, confidence region, catalytic reactor for aniline production

\section{Introduction}

The central equipment in a chemical plant is the chemical reactor, where the high value products are obtained. When the chemical reactor presents a high sensitivity to operating conditions, and additionally highly exothermic hazardous reactions are conducted, the reactor operation implies stability problems and risk of runaway. This is the reason why optimization efforts have to focus not only on production maximization (economic objectives), but also on minimizing the operating risk (safety, stability, controllability objectives). This optimization problem translates in a multi-objective optimization in the presence of multiple technological constraints. Besides, model-based setting of the optimal operating policy for such highly sensitive chemical reactors is a difficult task if the entire process complexity is accompanied by parametric uncertainties.

On the other hand, modelling the considered continuously operated chemical reactor implies to account for the system dynamics, including the chemical reactions and heat transfer from the reactor tubes to the cooling agent, which is time/ length-varying and of nonlinear characteristics. Additionally, the following process uncertainty sources have to be considered: model inaccuracies, variabil-

"professor, corresponding author; mail address: University POLITEHNICA of Bucharest, Chemical and Biochemical Engineering Department, P.O. 35-107 Bucharest Romania; Tel: +40 744830 308; E-mail: gmaria99m@hotmail.com

${ }^{\S}$ PhD student; leaving from Najaf Technical College Al-Furat Al-Awsat Technical University, Iraq. ity in raw-material purity and catalyst characteristics; the simplifying hypotheses used to represent the complex kinetics of the main and side reactions; a certain random fluctuation of the operating parameters/control variables around the set-point due to limited performances of the control system, etc.

When designing/operating a chemical reactor, where a process of high thermal sensitivity is conducted, there are a number of "sensitive" engineering issues which have to be addressed, that is:

- Minimize the reactor operating risk while keeping a maximum profit, which translates into determining the reactor optimal size and operating parameters/policy (setpoint) within technological constraints. The presence of uncertainties in the control variable runaway boundaries, and the parametric random disturbances, impose adoption of prudent operating solutions in spite of the temptation to drive the nominal running point in a vicinity of the runaway boundaries in the parametric space to get higher reactor productivity. Such a safe operation often means keeping the setpoint "outside the confidence region of the safety limits", by eventually over-designing the cooling/control system. ${ }^{1-6}$

- Determine the plant optimal operation policy at the maximum productivity (or at least within benefit limits) while minimizing the accident consequences scenario. ${ }^{7,8}$ It should be mentioned that the entire plant productivity depends on accident frequency and their effects/consequence magnitude. , $^{5,8}, 9$

- Consider various sources of uncertainties when solving the mentioned multi-objective optimization problem, e.g. uncertainty in the control vari- 
able runaway boundaries, and the inherent random disturbances of the control variable setpoint. ${ }^{5,8,9,32-36}$

On the other hand, the plant optimal operating policy should be considered as a trade-off between economic, environmental, and safety objectives. By using a new "safe operation criterion" introduced by Maria and Dan, ${ }^{10-14}$ based on the sum of two failure probability indices related to uncertainty in the reactor runaway boundaries and random disturbances in the operating parameters, a multi-objective optimization can be formulated by defining the sustainability by simultaneously considering technological, economic, and safety constraints.

A classical way to solve the above-mentioned optimization problem implies searching the optimal control variable set-point, usually related to the chemical reactor input variables (e.g. feed flow rate, feeding composition, cooling agent temperature, overall and species partial pressures) that ensure minimum/maximum values of an objective function (i.e. the performance objective, or 'cost' function in financial or engineering terms) while considering the mass, heat, and momentum equations together with the technological-safety constraints. ${ }^{4,15}$ For multi-objective optimization with contrary objectives, one elegant option is to obtain the set of Pareto optimal solutions, also called "Pareto-front" for the case of at least two adverse objectives. A Pareto solution is one where any improvement in one objective can only take place at the cost of another objective. ${ }^{37}$

According to Maria and Dan, the thermal runaway risk during reactor operation can be quantified, and included in the multi-criteria optimization function by using an overall failure probability index. ${ }^{10-14}$ Briefly, the methodology of Maria and $\operatorname{Dan}^{10-14}$ to obtain the Pareto-optimal operating policies for a multi-objective optimization problem in the presence of uncertainty in the control variables and runaway boundaries includes: derivation of the process and reactor dynamic model; definition of the technological constraints; evaluation of the safety limits and their confidence band for the control variables in the parametric space by also considering their random fluctuations around the setpoint; ${ }^{14}$ approximation of the critical variety with simple algebraic correlations to facilitate further reactor optimization rule; evaluation of the joint failure probability index based on the parametric and safety limit uncertainty, and then formulation of the multi-criteria objective function followed by derivation of the so-called "optimal-Pareto front" solution. Then, a trade-off optimal solution is selected based on several criteria.

The present study is aiming at exemplifying this reactor operation optimization methodology of Maria and Dan ${ }^{10-14}$ to obtain the Pareto-optimal op- erating policy for the case of an industrial fixed-bed tubular reactor used for the catalytic hydrogenation of nitrobenzene to aniline in vapor-phase in the presence of uncertainty in safety limits of the operating region and uncertainty associated with the random fluctuations of the control variables. The applied model-based methodology to obtain the Pareto-operating policies of the reactor is that of Maria and Dan. ${ }^{10-14}$ This methodology, described here very briefly, is detailed below in the Theory Section.

\section{Material and methods}

\section{Nitrobenzene hydrogenation process and reactor characteristics}

Aniline, produced worldwide in large quantities, is a precursor of many industrial chemicals. Under normal conditions, aniline is a non-inflammable liquid, of low water solubility and vapour pressure. It is highly toxic (by inhalation, ingestion, or percutaneous adsorption). Aniline production is mainly based on the catalytic hydrogenation of nitrobenzene (NB) at near normal pressures and 300$350{ }^{\circ} \mathrm{C}$ temperatures (for more details see the reaction and process conditions given in Table $1^{1,2}$ ).

Gaseous nitrobenzene catalytic hydrogenation is the principal industrial method for economic production of aniline. The exothermic process (reaction heat of $-106.01 \mathrm{kcal} \mathrm{mol}^{-1} \mathrm{NB}$ ) is carried out within a fixed-bed or a fluidized-bed reactor with high yields $\left(99 \%{ }^{16}\right)$, even though trials on a monolith reactor have also been investigated. ${ }^{17,18}$ The catalyst used is copper or palladium on an activated carbon or an oxidic support, sometimes modified by adding other metals $(\mathrm{Ni}, \mathrm{Pb})$ or promoters to increase the selectivity $\left(\mathrm{Mg}, \mathrm{Ca}, \mathrm{Zr}\right.$, Th, $\left.\mathrm{V}, \mathrm{Cr}^{19}\right)$. The process is conducted at temperature values within $250-350{ }^{\circ} \mathrm{C}$, low pressure values (below $10 \mathrm{~atm}$ ) but with a high excess of hydrogen (up to 1:100 - 1:200 molar NB/ $\mathrm{H}_{2}$ ) to make the NB conversion practically complete, and avoid large partial NB pressures leading to high generated reaction heat (risk limits being around 1:10 feed ratios $^{2}$ ). Under these conditions, the equilibrium of the main reaction:

$$
\mathrm{NB}+3 \mathrm{H}_{2} \underset{k^{\prime}}{\stackrel{k}{\longrightarrow}} \mathrm{A}+2 \mathrm{~W}
$$

is totally shifted towards aniline formation, the fugacity coefficient being very close to unity, while the equilibrium conversion is practically complete. ${ }^{20}$ The overall pressure rise favours the direct reaction, but can also induce critical conditions due to the inherent increase of NB partial pressure and generated heat of reaction.

The plant capacity of aniline production is placed in the range of $18,000-130,000 \mathrm{t} \mathrm{y}^{-1}$. The 
Table 1 - Tubular fixed-bed reactor model, catalyst and industrial reactor characteristics and nominal operating conditions for the nitrobenzene catalytic hydrogenation to aniline in vapour phase (ca. 48,000 t AN/y production capacity; adapted after ${ }^{l-3}$ )

Model hypotheses:

- ideal plug-flow with concentration, temperature, and pressure gradients in only axial direction, $\quad-$ for $L / d_{p}>50$ i.e. $\partial\left(c_{j}, T, p\right) / \partial R_{t}=0$

- negligible axial dispersion coefficient, i.e. $D_{z}=0$

- isothermal spherical catalyst particle, i.e. $\partial T_{p} / \partial R_{p}=0$

- negligible extragranular resistance to the transport of property (Satterfield criterion ${ }^{29}$ )

- intraparticle mass transport resistance expressed by the overall effectiveness factor $\eta=\frac{3}{\Phi}\left(\frac{1}{\operatorname{th}(\Phi)}-\frac{1}{\Phi}\right)$

- spherical particle with avg. diameter $d_{p}$

- for $L / d_{p}>100$

- $\Phi(z)$ evaluation using Table 1 formula

- heat transfer coefficient computed with the formula:

$\frac{1}{U}=\frac{1}{h_{i}}+\frac{\delta_{t}}{\lambda_{t}} \frac{A_{b}}{A_{m}}+\frac{1}{h_{e}} \frac{A_{b}}{A_{e}} ; \frac{h_{i} d_{t}}{\lambda_{g}}=3.5 \operatorname{Re}_{p}^{0.7} e^{-4.6 d_{p} / d_{d}} ;$

$-\operatorname{Re}_{p}=\left(\frac{d_{p} F_{m}}{\mu_{g} S_{t}}\right)$

(approximate value for nominal inlet conditions is $U=446 \mathrm{kcal} \mathrm{m}^{-2} \mathrm{~h}^{-1} \mathrm{~K}^{-1}$ )

- friction factor computed with the Ergun and Hicks formula:

$$
\begin{aligned}
& f=\frac{1-\varepsilon}{\varepsilon^{3}}\left[1.75+\frac{150(1-\varepsilon)}{\operatorname{Re}_{p}}\right], \text { for } \operatorname{Re}_{p} /(1-\varepsilon)<500 ; \\
& f=6.8 \frac{(1-\varepsilon)^{1.2}}{\varepsilon^{3}} \operatorname{Re}_{p}^{-0.2}, \text { for } \operatorname{Re}_{p} /(1-\varepsilon) \geq 500 .
\end{aligned}
$$

- Thiele modulus $\Phi$ is evaluated with the pseudo $n$-th kinetic order formula of footnotes (a-b) ${ }^{22}$. The effective diffusivity in the catalyst particle is: $D_{e f} \approx \frac{\varepsilon}{\tau} D_{m}$ (the surface and Knudsen
diffusivity contribution are neglected).

Reactor differential balance equations: Observations

mass balance: $F_{M, N B, 0} \frac{\mathrm{d} X_{N B}}{\mathrm{~d} z}=S_{t} \rho_{c} \eta r_{N B}$ At $z=0$; $X_{N B, 0}=0$;

heat balance: $\left(\sum_{j} F_{M, j} c_{p, j}\right) \frac{\mathrm{d} T}{\mathrm{~d} z}=(-\Delta H) S_{t} \rho_{c} \eta r_{N B}-U\left(\pi d_{t}\right)\left(T-T_{a}\right)$, $T=T_{0}$ $p=p_{0}$. $j=N B, H, A, W$

momentum balance: $\frac{\mathrm{d} p}{\mathrm{~d} z}=-f\left(\frac{F_{m}}{S_{t}}\right)^{2} \frac{1}{\rho_{g} d_{p}}$

$r_{N B}=k p_{N B}^{n} p_{H}^{m}, n=0.5 ; m=0.5$; see footnotes (c-d)

Nominal operating conditions:

Inlet overall pressure

Inlet gas temperature

Feed molar ratio (moles $\mathrm{H}_{2} /$ moles NB)

Feed NB flow-rate (per reactor tube)

Gas superficial velocity (related to void tube)

Cooling agent average temperature

Molar flow-rate variation according to the overall reaction stoichiometry:

$F_{M, N B}=F_{M, N B, 0}\left(1-X_{N B}\right) ; F_{M, H}=F_{M, N B, 0}\left(M-3 X_{N B}\right)$;

$F_{M, A}=F_{M, N B, 0} X_{N B} ; F_{M, W}=F_{M, N B, 0} 2 X_{N B} ; F_{M, t}=\sum_{j} F_{M, j}$.

$p_{0}=1.2 \mathrm{~atm}$

$T_{0}=573 \mathrm{~K}$

$M=15$

$F_{N B, 0}=1 \mathrm{~kg} \mathrm{~h}^{-1}$

$u_{0}=1.14 \mathrm{~m} \mathrm{~s}^{-1}$

$T_{a}=558 \mathrm{~K}$

$F_{m, t}=$

$F_{M, N B, 0}\left(1+M \frac{M_{H}}{M_{N B}}\right)$

Partial pressure is computed with the ideal gas formula: $p_{j}=p F_{M, j} / F_{M, t}$ 


\begin{tabular}{ll}
\hline Variable: & Observations: \\
\hline Catalyst density (bulk), $\rho_{c}$ & $1600 \mathrm{~kg} \mathrm{~m}^{-3}$ \\
\hline Catalyst particle average diameter, $d_{p}$ & $3 \mathrm{~mm}$ \\
\hline Catalyst porosity ${ }^{28}, \varepsilon$ & 0.5 \\
$\varepsilon=0.38+0.073\left[1+\frac{\left(d_{t} / d_{p}-2\right)^{2}}{\left(d_{t} / d_{p}\right)^{2}}\right]$ & \\
\hline
\end{tabular}

Catalyst tortuosity (adopted average value according to Satterfield ${ }^{29}$ ), $\tau \quad 4$

\begin{tabular}{ll}
\hline Reactor inner diameter, $d_{t}$ & $30 \mathrm{~mm}$ \\
\hline Reactor tube thickness, $\delta_{t}$ & $5 \mathrm{~mm}$ \\
\hline Reactor length, $L$ & $3 \mathrm{~m}$ \\
\hline Overall heat of reaction dependence with the temperature: & - using the data of ${ }^{2}$
\end{tabular}

$(-\Delta H)=-110604.0612+8.745102723 \cdot T-0.307037744$

$0.2776795668 \cdot 10^{-5} T^{3}+0.9639094601 \cdot 10^{-9} T^{4}-0.5970616340 \cdot 10^{-12} T^{5}$

[approximate value at nominal inlet conditions is $(-\Delta H)=106.01 \mathrm{kcal} \mathrm{mol}^{-1} \mathrm{NB}$ ].

Notes:
(a) The effectiveness factor is considered for a spherical catalyst particle of $d_{p}$ diameter, i.e.: $\eta=\frac{3}{\Phi}\left(\frac{1}{\operatorname{th}(\Phi)}-\frac{1}{\Phi}\right)$. For the NB

reaction rate $r=k p_{N B}^{n} p_{H}^{m}$, and large inlet ratios $M$, the Thiele modulus $\Phi$ is evaluated with the pseudo $n$-th kinetic order formula ${ }^{22}$ : $\Phi=\left(\frac{d_{p}}{6}\right) \sqrt{\left[\frac{n+1}{2}\left(k \rho_{p} \frac{1-\varepsilon}{\varepsilon} R_{g} T\right) p_{N B}^{n-1} p_{H}^{m}\right]_{\varepsilon} / D_{e f}}$. The effective diffusivity in the particle is approximated with the effective molecular diffusivity in the gas mixture $22: D_{e f} \approx \frac{\varepsilon}{\tau} D_{m}$ (the surface and Knudsen diffusivity contribution can be neglected here). The molecular diffusivity is computed with the Gilliland-Maxwell formula ${ }^{30}: D_{m}=4.3 \cdot 10^{-7} \frac{T^{1.5}}{p\left(v_{N B}^{0.33}+v_{H}^{0.33}\right)^{2}} \sqrt{\frac{1}{M_{N B}}}+\frac{1}{M_{H}},\left(\mathrm{~m}^{2} \mathrm{~s}^{-1}\right)$, where: $T=$ temperature $(\mathrm{K}) ; p=$ overall pressure $(\mathrm{atm}) ; v_{A}=$ molar diffusion volume of species A the normal boiling point $\left(\mathrm{cm}^{3} \mathrm{~mol}{ }^{-1} ; 3^{31}\right)$; $M_{A}=$ molecular mass of species $\mathrm{A}\left(\mathrm{g} \mathrm{mol}^{-1}\right) ; \varepsilon=$ catalyst porosity; $\tau=$ particle tortuosity.

(b) Thiele modulus $\Phi$ is evaluated with the pseudo $n$-th kinetic order formula, for cases with large values of $M^{22}$ :

$\Phi=\left(\frac{d_{p}}{6}\right) \sqrt{\left[\frac{n+1}{2}\left(k \rho_{p} \frac{1-\varepsilon}{\varepsilon} R T\right) \alpha p_{N B}^{n-1} p_{H}^{m}\right] / D_{e f}}$.

(c) The rate constants are ${ }^{20}: k=4.104 \cdot 10^{4},\left(\mathrm{kmol} \mathrm{kg}_{\text {cat }}{ }^{-1} \mathrm{~h}^{-1} \mathrm{~atm}^{-1}\right) ; E / R_{g}=8240 \mathrm{~K}$.

(d) $c_{N B}$ expressed in $\mathrm{kmol} \mathrm{L}^{-1} ; c_{j}=p_{j} /\left(R_{g} T\right)$.

very exothermic reaction (reaction enthalpy $=$ $106.01 \mathrm{kcal} \mathrm{mol}^{-1} \mathrm{NB}$ ) leads to a significant thermal hotspot in the catalytic bed in spite of intense cooling agent circulation in the outside tubes. This thermal hotspot is extremely sensitive to the operating conditions (i.e. inlet temperature, pressure, molar $\mathrm{H}_{2} / \mathrm{NB}$ ratio, gas flow-rate, and cooling agent temperature). The reactor output contains ca. $10 \%$ aniline, $70 \% \mathrm{H}_{2}$, and $20 \%$ water vapours (\%vol.) when it is operated close to the runaway boundaries. Maria and Stefan ${ }^{1-3}$ derived complex correlations of the critical operating conditions with the operating parameters.

A quite reduced number of systematic studies have been published reviewing the kinetic models proposed in the literature for this process (see e.g. the reviews ${ }^{2,21}$, most of the proposed models indi- cating apparent power-law type kinetics of fractional orders in NB and $\mathrm{H}_{2}\left(r=k p_{\mathrm{NB}}^{n} p_{\mathrm{H}_{2}}^{m}\right)$. Some studies developed mechanistic Langmuir-Hinshelwood hyperbolic type kinetic models, based on the NB and $\mathrm{H}_{2}$ absorption on the catalyst active sites, where the rate determining step is the surface reaction between the NB molecule and the $\mathrm{H}$ atom. ${ }^{17,19} \mathrm{~A}$ comparison between the apparent reaction rates predicted by various models under nominal inlet conditions of the industrial reactor has been made $\mathrm{in}^{2}$, revealing a quite different activity of various fresh catalysts, with rate values in the range of $0.007-0.4304$ $\mathrm{kmol} \mathrm{kg} \mathrm{kg}^{-1} \mathrm{~h}^{-1}$, for the same high particle effectiveness factor (usually $0.98-0.99$ ). With the increase in temperature over the reactor length, these discrepancies among various catalysts exacerbate, as revealed by the displayed reaction rates $i^{2}$ under adi- 
abatic operating conditions. The plotted temperature and NB conversion profiles in ${ }^{2}$, of sigmoid or monotonous curve shapes, suggest significant differences in reaction activation energy for various catalysts. Indeed, as reported in literature, the limiting rate step seems to be the hydrogen molecule activation on the Ni-catalyst, compared to the nitrobenzene molecule activation on the $\mathrm{Pt}$ or $\mathrm{Cu}$ catalyst surface. The catalyst deactivation is significant even for short times on stream, and several kinetic models have been proposed in literature, based on the coke formation mechanism. ${ }^{18,19}$ The catalyst activity decay has to be accounted for by reactor optimization via a time-dependent feeding policy. However, for the reactor risk analysis, the fresh-catalyst conditions are more interesting and correspond to a higher reactor parametric sensitivity, which will be used further in establishing the safety margins of the operating region.

The industrial fixed-bed reactor constructive solution, as described by Trambouze et al., ${ }^{22}$ attempts to remove quickly the reaction heat generated inside the large number of tubes filled with the catalyst, by means of a good circulation of the cooling liquid in which the tubes are immersed. The heat transfer-limiting step is located on the solid catalyst side. Various constructive versions are reported to ensure a high productivity and good heat release, even if the close temperature control remains a critical issue (e.g. $X_{\mathrm{NB}}=0.999$ under a $\mathrm{Cu}-\mathrm{Mg}$-silica catalyst and large time-on-stream for $553-573 \mathrm{~K}, M=17$, and $F_{t}=0.35 \mathrm{~kg} \mathrm{~kg}_{\text {cat }}{ }^{-1} \mathrm{~h}^{-1}$; review of ${ }^{23}$ ).

A relatively simple reactor model, of pseudo-homogeneous type, has been adopted in this paper in order to perform the risk analysis, without radial gradients and of plug-flow fluid circulation. ${ }^{24}$ The considered mass, heat, and momentum ordinary differential equation (ODE) balances assume the hypotheses mentioned in Table 1, together with the catalyst characteristics, and the intra-particle mass transfer resistance through an isothermal effectiveness factor (Table 1 footnote). The model assumes a constant temperature of the cooling agent and overall heat transfer coefficient, and the intra-particle mass transfer resistance through an isothermal effectiveness factor. The gas properties are approximated with those of the air due to the NB small fraction (lower than $9 \%$ mol.). The industrial tubular reactor has a length $L=3 \mathrm{~m}$, and an inner diameter $d_{t}=30 \mathrm{~mm}$.

The main variables of the model and the nominal stationary operating conditions of the reactor are presented in Table 1. The reactor model has been solved with a low-order stiff ODE integrator to account for cases of using catalysts displaying higher activation energies. It is of interest to quantitatively characterize the potential hazard of the main chemical reaction before starting the reactor sensitivity analysis. Some reaction risk indices under adiabatic conditions are presented by Maria and Stefan ${ }^{2}$ (for an extensive discussion see ${ }^{7,25}$ ). The very exothermic reaction leads to a temperature rise under adiabatic conditions of $\Delta T_{a d}=(-\Delta H) c_{j, 0} /\left(\bar{\rho} \bar{c}_{p}\right), c_{j, 0}=$ initial concentration of key species; $\bar{\rho}=$ reacting mixture density; $\bar{c}_{p}=$ average specific heat ${ }^{26}$ of $697.95 \mathrm{~K}$, which largely outruns the limit of $50 \mathrm{~K}$ recommended for out-of-risk primary reactions. ${ }^{25}$ The resulting temperature increase $\Delta T_{\text {peak }}=T_{\max }-T_{0}$ in the non-adiabatic reactor depends on the catalyst and operating conditions, and it can easily reach more than $100 \mathrm{~K}$ for certain fresh catalysts. One rough measure of reaction hazard is given by the reaction violence index $B=\Delta T_{\mathrm{ad}} E R_{\mathrm{g}}^{-1} T_{0}{ }^{-2},{ }^{25}$ where $\Delta T_{\text {ad }}$ is the temperature rise under adiabatic conditions; $E$ is the activation energy, and $T_{0}$ is the inlet temperature. For dangerous reactions, the reaction violence index $B$ is larger than the threshold $B=5$, especially for catalysts exhibiting high activation energies (as in the studied case) ${ }^{25}$. Low values obtained for $T M R_{a d}$ (reaction time-to-maximum rate under adiabatic conditions $)^{12}$ correspond to a risky reaction, but also to short length-to-maximum- rate values under adiabatic conditions, often less than $0.1 \mathrm{~m}$ within the reactor. Such a result indicates the reactor section near the inlet point as presenting the highest thermal sensitivity to parametric conditions.

Since the thermal sensitivity is one of the most important issues to be considered during reactor optimization, the fixed-bed constructive alternative employing thin tubes ${ }^{11}$ is intended to rapidly dissipate the generated heat as fast as possible. The tubes filled with catalyst are immersed in the cooling agent, which rapidly circulates across the tubes, efficiently taking over the reaction heat. Still, the limiting step of the heat transfer is located on the catalyst side, which means that the thermal control needs to address the inlet conditions rather than the cooling agent. The parametric sensitivity regime of this process under nominal conditions corresponds to slow reactions, ${ }^{1,2}$ with an effectiveness factor $(\eta)$ for the solid particle very close to 1 (catalyst characteristics are given in the Table 1). For optimization and risk analysis purposes, a simple kinetic model was adopted.

The analysis starts with determining the most influential parameters in respect to the process performance (conversion, yield), which were found $d^{1,2}$ to be (generically denoted by control variable vector $\boldsymbol{u}$ ): the cooling agent absolute temperature $T a$, the inlet butane molar fraction $y_{B u, 0}$, the inlet pressure $p_{0}$, and the absolute inlet temperature $T_{0}$. 
Theory: Derivation of the safety criteria and the Pareto-optimal front of reactor operating policies

The next step of the sensitivity analysis involves evaluating the safety limits for the control variables $\boldsymbol{u}(\phi)$ in the parametric space $(\phi)$, by using the generalized sensitivity criterion of Morbidelli and $\operatorname{Varma}^{5}$ ( $\mathrm{see}^{2}$ for the computational details).

The procedure is as follows: Firstly it is necessary to determine the runaway boundaries of the control variables in successive 2D parametric planes by using the generalized sensitivity criterion of Morbidelli and Varma ${ }^{5}$ (MV method). Sensitivity-based methods are based on determining the parametric sensitivity functions of state variables $y_{i}$ with respect to parameters $\varphi_{i}$, in absolute or relative terms:

$$
\begin{aligned}
& s\left(y_{i} ; \varphi_{j}\right)=\partial y_{i} / \partial \varphi_{j} ; S\left(y_{i} ; \varphi_{j}\right)=\left(\varphi_{j}^{*} / y_{i}^{*}\right) s\left(y_{i} ; \varphi_{j}\right)(1) \\
& \text { (referring to a nominal operating point } \left.\phi_{j}{ }^{*}\right) .
\end{aligned}
$$

The parametric sensitivity analysis highlights the major influence on the hotspot [i.e. temperature peak $\left.\Delta T_{\text {peak }}=\left(T_{\max }-T_{0}\right)\right]$ of the inlet NB partial pressure $p_{\mathrm{NB}, 0}$ (via the overall pressure $p_{0}$, and $\mathrm{M}$ the inlet $\mathrm{H}_{2} / \mathrm{NB}$ ratio), the cooling fluid temperature $\left(T_{a}\right)$ circulated in the extra-tubular space, and the inlet gas temperature $\left(T_{0}\right)$. Under certain critical inlet conditions $\left(p_{0 c}, T_{0 \mathrm{c}}\right)$, to be determined for every catalyst, the hotspot rises sharply and the reactor hotspot operation evolves towards pseudo-adiabatic conditions and eventually process runaway. The dependent variables of most interest for the safety and economic analysis are $\Delta T_{\text {peak }}\left(T_{\text {max }}\right)$ and the outlet NB conversion $X_{\mathrm{NB}}(\mathrm{L})$.

The state $\boldsymbol{y}$ sensitivity functions $S(\boldsymbol{y} ; \phi)_{z}$ (dependent on the $z$-reactor length) have been computed by means of a finite difference numerical method, involving repeated simulations of the reactor behaviour under various conditions, every time solving the mass, heat, and momentum differential balance equation of the reactor model. This z-MV criterion (referring the state sensitivity functions varying with the reactor length $z$ ) has been reported as being robust to complex kinetic models, indicating less conservative predictions of runaway boundaries than most geometrical or explicit methods. ${ }^{5,27}$ The runaway boundary of the control variable $u(\varphi)$ corresponds in the MV method to the Max $\left|S\left(T_{\max } ; u\right)\right|$. Thus, by taking $u=T_{0}$, the resulting $\left[S\left(T_{\max } ; T_{0}\right)\right.$ vs. $\left.T_{0}\right]$ plots for various $\varphi_{j}$ operating parameter values, allow establishing the runaway boundaries in every parametric plane $\left[T_{0}\right.$ vs. $\left.\varphi_{j}\right]$, as represented in Fig. 1(a) for $\Phi_{j}=T_{a}$.

In the next step, to reduce the computational effort during the optimization analysis and safety index evaluation, simple adequate algebraic correla- tions of the control variable safety limits $u_{i . c}(\phi)$ and of their variance $\sigma_{u_{j c}}^{2}$ can be derived, usually in logarithmic or polynomial form,,$^{1-3,10-14}$ from bringing together all critical curves obtained by means of the MV criterion, that is for the aniline reactor present case: ${ }^{1}$

$$
\begin{aligned}
T_{0 c}= & -2277.6489-93.1459 \cdot p_{0}+14.4202 \cdot M+ \\
& 11.6506 \cdot T_{a}-0.2554 \cdot M^{2}-0.0116 \cdot T_{a}^{2} \\
p_{0 c}= & 115.0318+0.1057 \cdot M-0.3746 \cdot T_{a}+0.0003 \cdot T_{a}^{2}
\end{aligned}
$$

where: $T_{0 c}=$ critical value of inlet gas temperature $T_{0}, p_{0 c}=$ critical value of inlet gas pressure; $M=$ molar fed ratio (moles $\mathrm{H}_{2} / \mathrm{NB}$ ); $T_{a}=$ average temperature of the external cooling agent.

A very important aspect considered in the present study is related to the uncertainty in evaluating the safety limits of the operating region associated with the random fluctuations $\left(\delta \varphi_{j}\right)$ in the parameters around the nominal set-point, i.e. $\varphi_{j} \pm \delta \varphi_{j}$. By repeatedly applying the MV-sensitivity method, while considering the parameters at lower or upper bounds, the lower and upper bounds of the critical conditions (the confidence region) can be thus obtained. The dispersion of such critical curves $u_{j, c}$ $(\phi)$ can be estimated by using the error propagation formula for the assumed uncorrelated parameters: ${ }^{28}$

$$
\begin{aligned}
\sigma_{u_{c}}^{2} & =\sum_{j}\left(\partial u_{c} / \partial \phi_{j}\right)_{\bar{\phi}}^{2} \cdot \sigma_{\phi_{j}}^{2}, \\
\text { with } \phi & =\left[T_{0}, T_{\mathrm{a}}, p_{0}, \mathrm{y}_{\mathrm{NB}, 0} \text { or } M\right],
\end{aligned}
$$

Such a statistical model is useful not only for quickly predicting the safety limits for operating parameters, but also allows a quick determination of their uncertainty. For instance, the standard deviation of the $T_{0 c}$ (denoted by $\sigma_{T T_{0}}$ ) can be evaluated approximately by using the error propagation formula for the assumed uncorrelated parameters, as follows:

$$
\sigma_{T_{0 c}}^{2}=\sum_{j}\left(\frac{\partial T_{0 c}}{\partial \varphi_{j}}\right)^{2} \sigma_{\varphi_{j}}^{2} ; \varphi_{j}=M, T_{a}, p_{0}, \ldots
$$

For the nominal conditions presented in Table 1 , the following parameter/control variable standard deviations of the control variable random fluctuations are considered: $\sigma_{y_{N B, 0}}=0.02 ; \sigma_{v M}=2 \mathrm{~mol} \mathrm{~mol}^{-1}$; $\sigma_{T_{0}}=10 \mathrm{~K} ; \sigma_{T_{a}}=10 \mathrm{~K} ; \sigma_{p_{0}}=0.2 \mathrm{~atm}$.

\section{Results}

\section{Evaluate the joint failure probability index under parametric and safety limit uncertainty}

During the reactor optimal set-point search, it is possible to determine, following the above procedure for each proposed parameter vector with known 

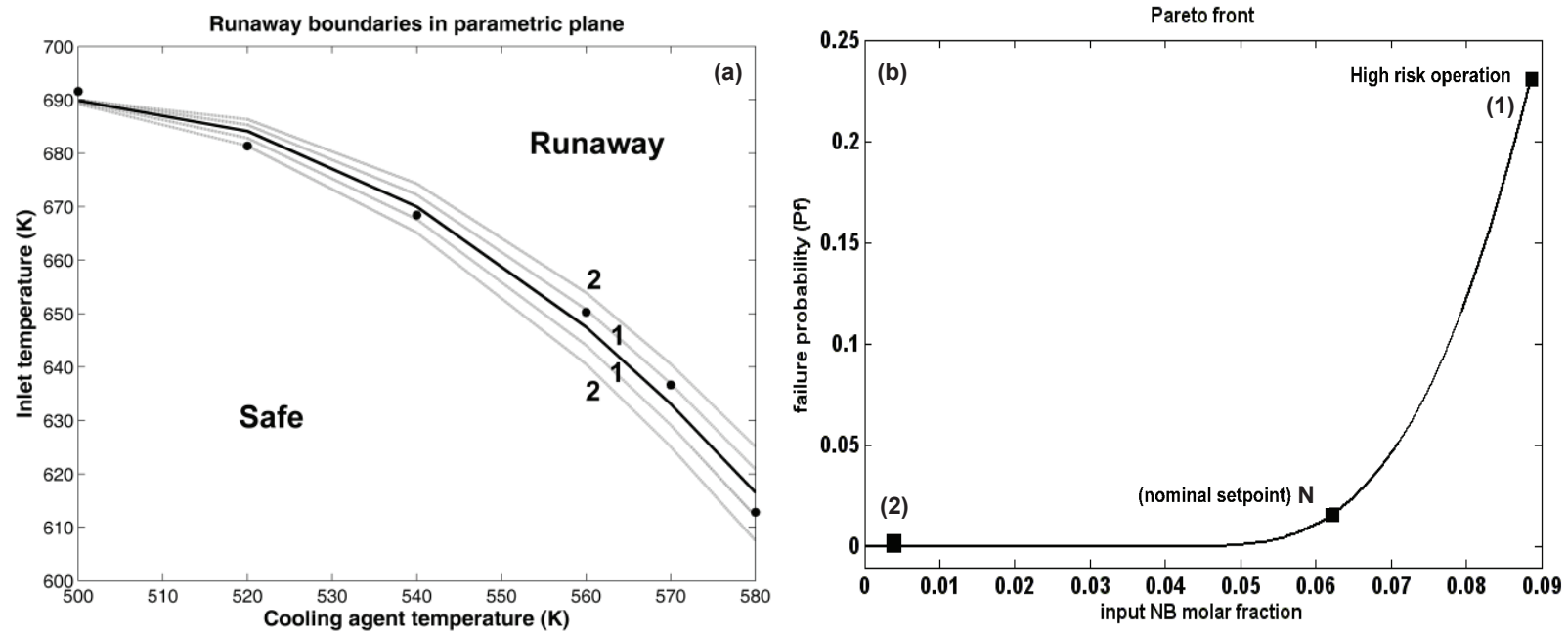

Fig. 1 - (a) Runaway boundaries ( ـ in the parametric plane $\left[T_{0} v s . T_{a}\right]$ and its confidence band for parametric deviations $\phi_{j} \pm \delta \phi$ of $\delta P_{0}=0.2 \mathrm{~atm}(1--), \delta P_{0}=0.4 \mathrm{~atm}(2---), \delta M=1.5 \mathrm{~mol} \mathrm{~mol}^{-1}(1--), \delta M=2 \mathrm{~mol} \mathrm{~mol}^{-1}(2---), \delta T=2 \mathrm{~K}(1---), \delta T=5 \mathrm{~K}(2---)$ (adapted following ${ }^{l}$ (b) Optimal Pareto operating policies for the tubular reactor, and some selected setpoints: nominal conditions of average runaway risk (N); operation of high risk-high productivity (1); operation of low risk-low productivity (2); Considered standard deviations of the control variable random fluctuations are $\sigma_{y_{N B, 0}}=0.02 ; \sigma_{M}=2 \mathrm{~mol} \mathrm{~mol}^{-1} ; \sigma_{T_{0}}=10 \mathrm{~K} ; \sigma_{T_{a}}=10 \mathrm{~K} ; \sigma_{p_{0}}=0.2 \mathrm{~atm}$.

parameter standard deviations, the control variable runaway limit and its associated standard deviation $\sigma_{u_{c}}$. Thus, a safety criterion can also be included in the optimization procedure, i.e., minimization of a runaway probability $P_{f}$ (which varies in the range of $[0,1])$. Following the definition of Maria and $\operatorname{Dan}^{10,14}$, this objective function includes the double parametric and safety limit uncertainty in only one cumulative index. The evaluation of the runaway risk probability has been proposed by Maria and $\operatorname{Dan}^{10,14}$ in the form of:

$$
P_{f}=P_{f 1}+P_{f 2}
$$

where:

i) the joint failure probability $P_{f 1}$ expresses the chance that the considered control variables will overpass the runaway boundaries as a consequence of the uncertainty in the safe operation limits, i.e.: $u_{i}(\boldsymbol{\varphi}) \geq \bar{u}_{i c}(\phi) \pm \delta u_{j c}(\phi, \delta \phi)$ for normal distributed runaway boundary $u_{j, c}^{j, c} \sim N\left(\bar{u}_{j, c}(\phi), \sigma_{u_{j, c}}^{2}\right)$, and operating parameters $\varphi_{j} \sim N\left(\bar{\varphi}_{j}, \sigma_{\varphi_{j}}^{2}\right)$;

ii) the joint failure probability $P_{f 2}$ reflects the chance that $\bar{u}_{j} \geq u_{i}(\phi)$ during operation due to the randomly (normally) fluctuating control variables $u_{j, c} \sim N\left(\bar{u}_{j}, \sigma_{u_{j}}^{2}\right)$, i.e.:

$$
\begin{aligned}
& P_{f 1}=\sum_{j} P_{N}\left(\bar{u}_{j, c}-d_{j}<u_{j, c}<\bar{u}_{j, c}+d_{j}\right) ; \\
& d_{j}=\left|u_{j}(\varphi)-\bar{u}_{j, c}(\varphi)\right| ; P_{f 2}=\sum_{j} P_{f 2, j} ; \\
& P_{f 2, j}=P_{N}\left(\bar{u}_{j}-d_{j}<u_{j}<\bar{u}_{j}+d_{j}\right),
\end{aligned}
$$

(where $P_{N}$ denotes the normal cumulative distribution function of the random variable). An overall joint failure probability results in the form $P_{f}=$ $P_{f 1}+P_{f 2}$, equivalent to the 'failure surface' in the 'most probable failure point' theory. ${ }^{10-15}$

In the considered case study, the parameter vector is $\phi=\left[M, T_{a}, P_{0}\right]$, while the control variables are

$$
\boldsymbol{u}=\left[T_{0}\left({ }^{\circ} \mathrm{C}\right), p_{0}(\mathrm{~atm}), M\left(\text { or } y_{N B O}\right), T_{a}\left({ }^{\circ} \mathrm{C}\right)\right] .
$$

\section{Reactor optimization problem formulation and result discussion}

An elegant and effective procedure for the multi-objective reactor optimization is to obtain the set of Pareto optimal solutions, also called Pareto-front for the case of two adverse objectives. A Pareto solution is one where any improvement in one objective can only take place at the cost of another objective. For continuous variables, an infinity of Pareto-optimal solutions might exist, and the final solution choice is subjective and case-dependent.

In the present study, two contrary objectives are considered, i.e. an economic objective related to reactor productivity vs. safety objective related to runaway failure probability, in the following formulation:

$$
\left[\hat{\boldsymbol{u}}_{0}, \hat{\boldsymbol{\varphi}}\right]=\arg \operatorname{Max}\left[y_{N B, 0}\right] \wedge \arg \operatorname{Min}\left[P_{f}\right]
$$

subjected to the following technological constraints: 


$$
\begin{gathered}
300<T_{0}<350\left({ }^{\circ} \mathrm{C}\right) \\
1.1<p_{0}<1.8(\mathrm{~atm}) \\
0.024<y_{\mathrm{NB}, 0}<0.095(\text { molar fr. }) \\
220<T_{\mathrm{a}}<350\left({ }^{\circ} \mathrm{C}\right) \\
T_{\max }<550\left({ }^{\circ} \mathrm{C}\right)
\end{gathered}
$$

When two opposite optimization criteria are used, an infinity of Pareto-optimal operating solutions can be found for the tubular reactor, each one corresponding to certain criteria trade-off in terms of manipulated / control variables $\boldsymbol{u}_{0}=\left[T_{0}, p_{0}, M, T_{a}\right]$, i.e.,: inlet temperature $\left(T_{0}\right)$, inlet overall pressure $\left(p_{0}\right)$, inlet molar fraction of NB $\left(y_{\mathrm{NB}, 0}\right)$ or, equivalently, $\mathrm{M}$ the inlet $\mathrm{H}_{2} / \mathrm{NB}$ molar ratio, and the cooling agent temperature $\left(T_{a}\right)$. The technological bounds of $\boldsymbol{u}_{0}$ are given in eq. (8). Uncertainties during optimization refer to the random fluctuations in the control variable setpoint, and are given below eq. (4) in terms of standard deviations.

The applied genetic algorithm implemented in Matlab ${ }^{\text {TM }}$ ("gamultiobj" routine) leads to obtaining the Pareto optimal front of the previous mathematical formulation at the expense of a quite significant computing time (dozens of minutes on a common
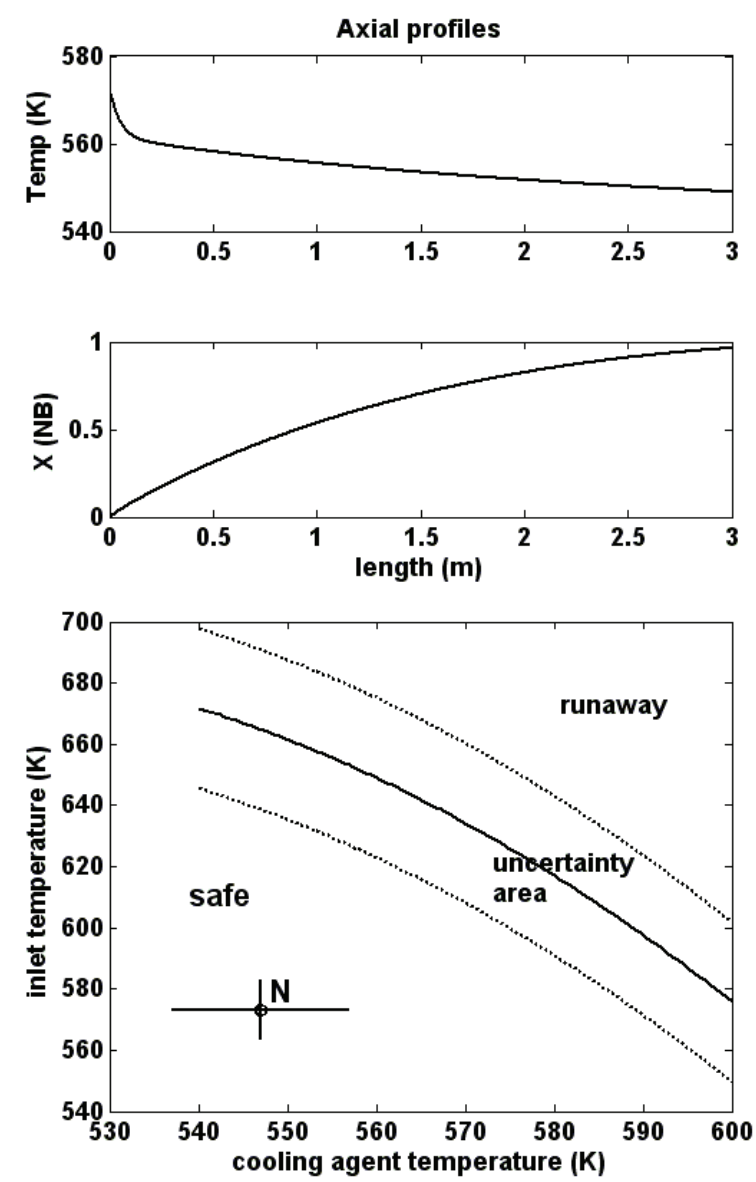

PC). An examination of the resulting Pareto optimal front plotted in Fig. 1(b) leads to the following conclusions:

i) To better interpret the Pareto front results, the location of the corresponding solution in the parametric space should be concomitantly investigated. This allows setting the selected reactor operation in the safety region, without crossing the confidence band of the safety limits (Fig. 1a).

ii) To illustrate the set point choice, three operating solutions have been selected from the Pareto front, as follows:

- The nominal operating point " $N$ ", corresponding to the nominal reasonably high reactor productivity in aniline (i.e. for $6.264 \%$ inlet molar fraction of NB), and of a reasonably small runaway risk of $1.7 \%$. This setpoint " $\mathrm{N}$ " corresponds to the following operating parameters:

$(\mathrm{N}):\left[T_{0}, p_{0}, y_{\mathrm{NB}, 0}, T_{a}\right]=\left[300^{\circ} \mathrm{C}, 1.2 \mathrm{~atm}\right.$, $\left.0.06264,274{ }^{\circ} \mathrm{C}\right], P_{\mathrm{f}}=1.7234 \%$;

$y_{\mathrm{NB}, 0}=0.06264$ corresponds to

$\mathrm{M} \stackrel{\mathrm{NB}, 0}{=} 1 / y_{\mathrm{NB}, 0}-1=15$ molar $\mathrm{H}_{2} / \mathrm{NB}$ inlet ratio.
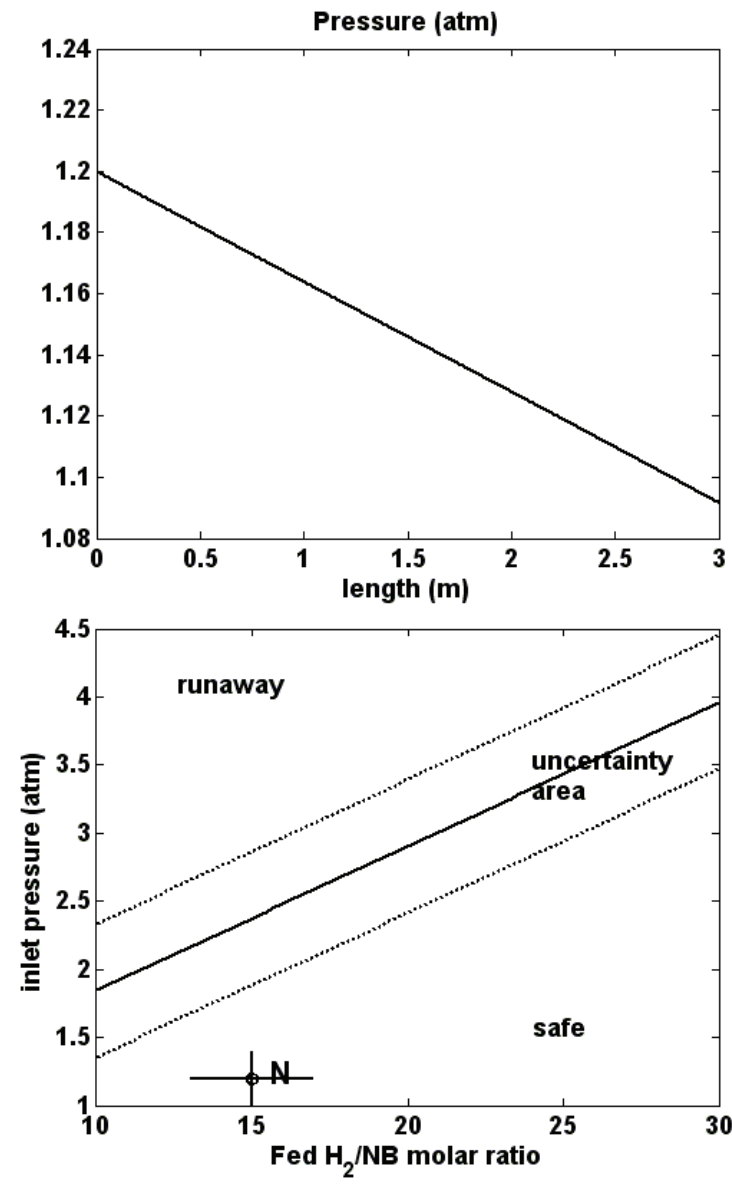

Fig. 2 - (Up-row) Axial profiles of the temperature, overall pressure, and conversion in the catalytic reactor for the nominal setpoint " $N$ " of Fig. 1 and Table 1. (Down-row) Location of the nominal setpoint " $N$ " and its uncertainty range in the [ $\left.T_{0} v s T_{a}\right]$ operating parameter plane (left) and in the $\left[p_{0} v s M\right]$ plane (right) compared to the runaway boundaries (solid lines) and their $68 \%$ confidence bands (dashed lines). 

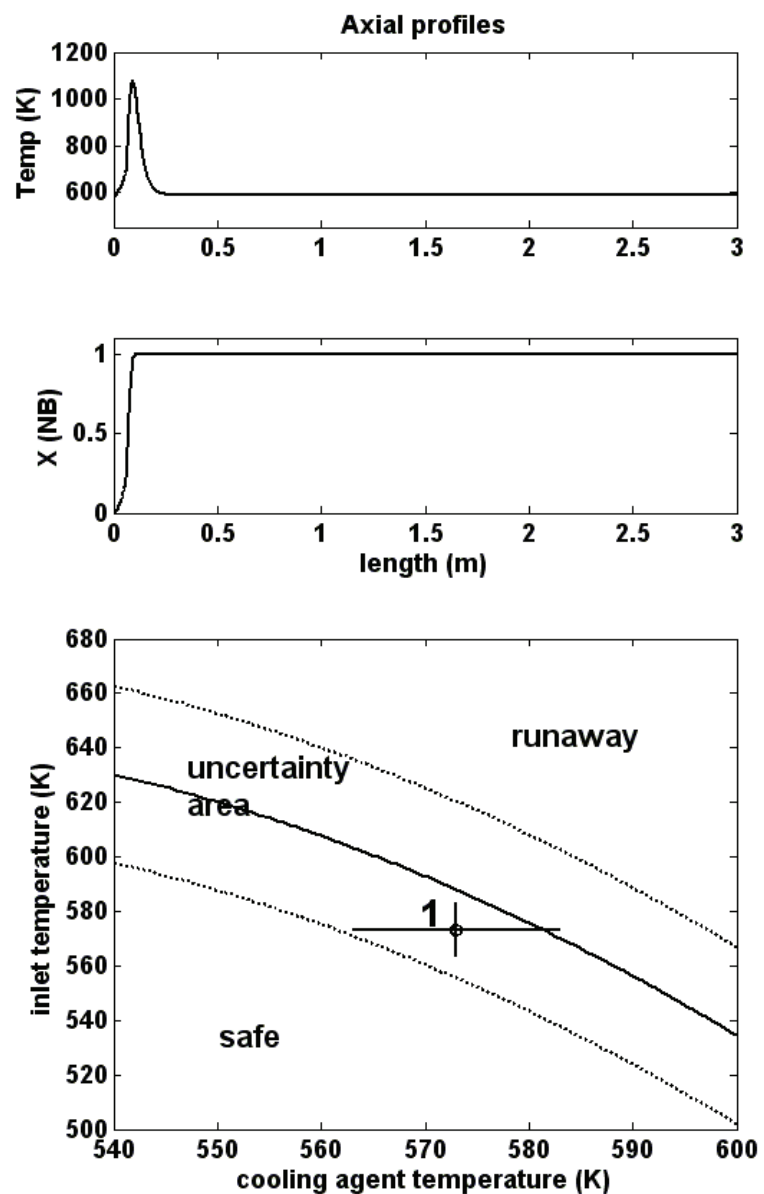
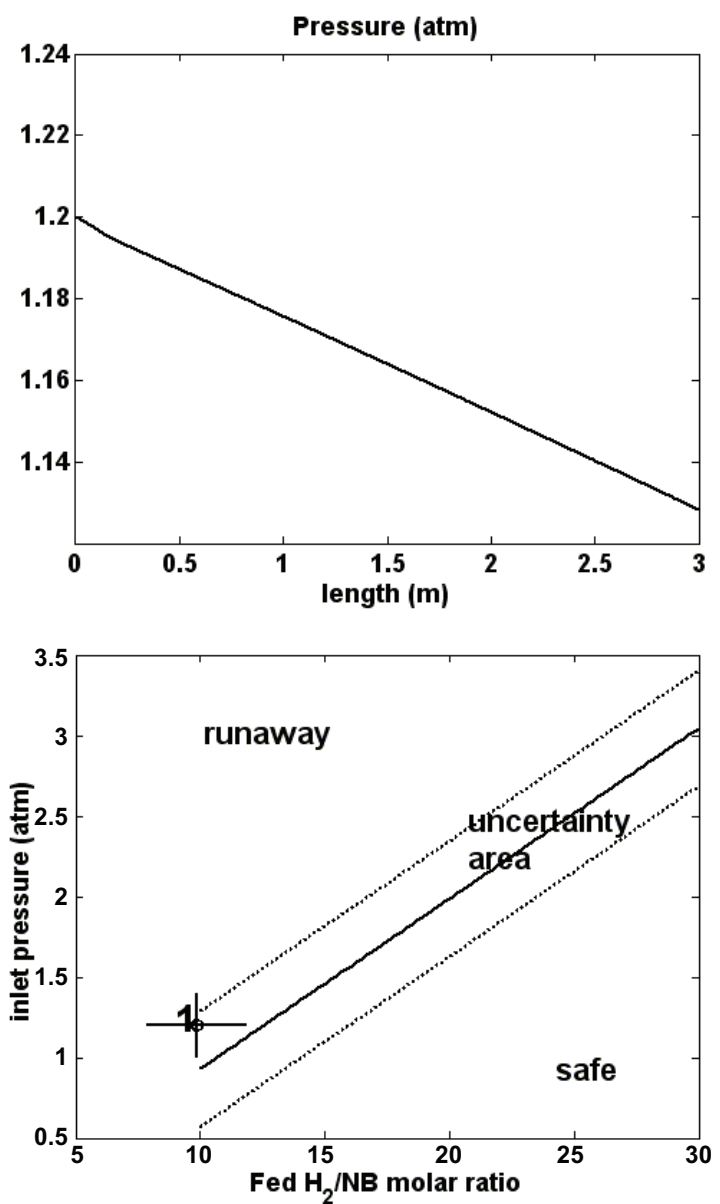

Fig. 3 - (Up-row) Axial profiles of the temperature, overall pressure, and conversion in the catalytic reactor for the nominal setpoint of high-risk "I" of Fig. 1. (Down-row) Location of the setpoint of high-risk " 1 " and its uncertainty range in the [T $v s T]$ operating parameter plane (left) and in the $\left[p_{0} v s \mathrm{M}\right]$ plane (right) compared to the runaway boundaries (solid lines) and their $68 \%$ confidence bands (dashed lines).

By simulating the reactor operation for the setpoint "N" conditions, and by plotting in Fig. 2 the axial profiles of the state variables in the reactor, together with the setpoint location in the $\left[T_{0}\right.$ vs $\left.T_{a}\right]$, and in the $\left[p_{0}\right.$ vs M] operating parameter planes, there are some conclusions to be derived: the reactor operation is stable with no temperature hot spot; the setpoint in the parametric plane is located very far from the runaway boundaries and their $68 \%$ confidence bands, being no risk of stepping over them due to random fluctuations in the setpoint control variables.

- The operating point " 1 " corresponding to a large AN productivity, i.e., 1.4-times larger than the nominal one, (i.e. for 0.092 inlet molar fraction of NB), but with the cost of a high runaway risk of $23 \%$. This high-risk setpoint "1" corresponds to the following operating parameters:

(1): $\left[T_{0}, p_{0}, \mathrm{y}_{\mathrm{NB} .0}, T_{a}\right]=\left[300{ }^{\circ} \mathrm{C}, 1.2 \mathrm{~atm}, 0.092\right.$, $\left.300{ }^{\circ} \mathrm{C}\right], P_{f}=23.066 \%$

$y_{\mathrm{NB}, 0}=0.092$ corresponds to $\mathrm{M}=1 / y_{\mathrm{NB}, 0}-1=10$ molar $\mathrm{H}_{2} / \mathrm{NB}$ inlet ratio.
By simulating the reactor operation for the setpoint " 1 " conditions, and by plotting in Fig. 3 the axial profiles of the state variables in the reactor, together with the setpoint location in the $\left[T_{0}\right.$ vs $\left.T_{a}\right]$, and in the $\left[p_{0}\right.$ vs $\left.\mathrm{M}\right]$ operating parameter planes, there are some conclusions to be derived: the reactor operation is unstable due to the presence of a high temperature hot spot (of ca. 1000 $\mathrm{K}$ ), while the setpoint in the parametric plane is located very close to the runaway boundaries and in the $68 \%$ confidence bands of the runaway boundaries, being of very high risk of process runaway even for small random fluctuations in the setpoint control variables, i.e., smaller than their standard deviations.

Consequently, in spite of a high AN productivity, the operating point " 1 " cannot be selected as a feasible setpoint.

- The operating point " 2 " corresponding to a large AN productivity, that is of 1.15-times higher than the nominal one, (i.e. for $7.176 \%$ inlet molar fraction of NB), with a slight increase in the runaway risk, i.e. up to $5.6 \%$. 
(2): $\left[T_{0}, p_{0}, y_{\mathrm{NB}, 0}, \mathrm{~T}_{\mathrm{a}}\right]=\left[300{ }^{\circ} \mathrm{C}, 1.2 \mathrm{~atm}, 0.07176\right.$, $\left.274{ }^{\circ} \mathrm{C}\right], P_{f}=5.5934 \%$

$y_{\mathrm{NB}, 0}=0.092$ corresponds to $\mathrm{M}=1 / y_{\mathrm{NB}, 0}-1=13$ molar $\mathrm{H}_{2} / \mathrm{NB}$ inlet ratio.

Reactor operation in the setpoint "2" conditions is stable; the setpoint in the parametric plane is located very far from the runaway boundaries and their $68 \%$ confidence bands, being of no risk to process runaway due to random fluctuations in the setpoint control variables.

Consequently, for highly sensitive reactors, subjective selection of the optimal operating policy (setpoint) by only using the Pareto-front plot, without considering the setpoint location in the parametric space, and its distance to runaway boundaries and their $68 \%$ confidence bands, is risky. The setpoint choice is even more difficult when any clear "break-point" in the Pareto front has been identified (where a sharp increase in the failure probability occurrs at a small rise in the productivity goal).

iii) The results obtained using the Pareto front indicate three reactor operating regions (Fig. 1b): a region close to the nominal point of a reasonable productivity and assumed small risk $(\mathrm{N})$; high productivity but of very high risk reactor operating region near the setpoint (1); a small productivity but of small risk reactor operating region near the setpoint (2).

iv) The optimal control variables must be kept far away from the runaway boundaries $u_{c}(\phi)$, but also from their confidence region $u_{c}(\phi) \pm \sigma_{u_{c}}$ in order to obtain prudent operation of the sensitive tubular reactor.

v) The operating alternatives can be drastically reduced if a supplementary criterion is considered during optimization [of economic or environmental nature, e.g. costs of the simulated accident consequences for various failure scenarios]. ${ }^{8,9}$

\section{Conclusions}

Choosing a multi-objective optimal operating policy for a chemical reactor is a difficult task. Although different optimization methods can be applied in order to mathematically determine the problem solution locus, the final decision depends on subjective priorities, and the parametric/model uncertainty level.

The applied model-based methodology of Maria and Dan ${ }^{10-14}$ to obtain an optimal operating policy for the studied tubular reactor accounts for an economic but also a probabilistic risk index. The procedure, even if computationally intensive, was proven simple and robust, being easily implementable on a common computer.

The selected multi-objective optimal operating policy of the reactor (the so-called Pareto front) is in fact a trade-off between opposite economic (reactor productivity) and safety criteria, but also accounts for the technological constraints, the safety boundary uncertainty, and the random fluctuations in the control variables. As a conclusion, the recommended optimal operation must be rather focused on more prudent operating conditions, searching for running points where the parameter disturbances do not lead to crossing the confidence region of the safety limits for all the control and operating variables, while keeping a reasonably small runaway probability (below $10 \%$ in the studied case).

\section{ACKNOWLEDGMENT}

The second author is grateful for the PhD study scholarship offered with the generosity of the Ministry of Education of Iraq.

\section{Notations list}

$B \quad$ - reaction violence index

$c_{j} \quad-$ molar concentration of species $j$

$c_{p^{\prime} j} \quad-$ molar specific heat of gaseous species $j$

$c_{p g} \quad-$ average specific heat of gas

$d_{p} \quad-$ particle diameter

$d_{t} \quad-$ reactor tube inner diameter

$D_{e f} \quad-$ effective diffusivity in the particle

$D_{m}, D_{j} \quad-$ molecular diffusivity of species $j$

E - activation energy

$F_{m} \quad-$ mass flow rate

$F_{M} \quad-$ molar flow rate

$f \quad-$ friction factor

$\boldsymbol{G}-$ Green's function matrix of the system

g $\quad-$ model function vector

$-\Delta H \quad-$ heat of reaction (at local temperature)

$h_{f p} \quad-$ gas-particle heat transfer coefficient

$\boldsymbol{J}$ - Jacobian matrix of the system

$k \quad-$ kinetic constant

$k_{f p} \quad-$ gas-particle mass transfer coefficient

$L \quad$ - reactor length

$M \quad-$ molar fed ratio (moles $\mathrm{H}_{2} / \mathrm{NB}$ ), or molecular weight

$n, m \quad-$ partial orders of reaction

$p \quad-$ overall pressure

$p_{0 c} \quad-$ critical inlet pressure

$p_{j} \quad$ - partial pressure of gaseous component $j$

$r \quad-$ chemical reaction rate 


$$
\begin{aligned}
& R_{g} \quad-\text { universal gas constant } \\
& S_{t} \quad-\text { tubular reactor cross-section } \\
& s(y ; \phi) \quad-\text { absolute sensitivity, } \partial y(z) / \partial \phi \\
& S(y ; \phi) \quad-\text { normalized sensitivity, }\left(\phi^{*} / y^{*}\right) s(y ; \phi) \\
& T \quad-\text { temperature } \\
& T_{0} \quad-\text { inlet temperature } \\
& T_{a} \quad-\text { average temperature of the external cooling } \\
& \text { agent } \\
& T_{0 \mathrm{c}} \quad-\text { critical inlet temperature } \\
& T_{\max } \quad-\text { maximum temperature in the reactor } \\
& T M R_{a d} \text { - time to maximum rate under adiabatic condi- } \\
& \text { tions } \\
& \Delta T_{a d} \quad \text { - adiabatic temperature rise } \\
& t \quad-\text { time } \\
& U \quad-\text { overall heat transfer coefficient } \\
& u, u_{0} \quad-\text { control variable, or gas superficial velocity } \\
& X_{j} \quad-\text { reactant } j \text { conversion } \\
& y, y_{j} \quad-\text { state variable, or molar fraction of gas com- } \\
& \text { ponent } j \\
& z \quad-\text { reactor axial coordinate } \\
& \Delta \quad-\text { finite difference } \\
& \varepsilon \quad-\text { catalyst porosity } \\
& \delta \quad-\text { random variation } \\
& \phi \quad-\text { operating parameter } \\
& \eta \quad-\text { effectiveness factor for solid particle } \\
& \lambda_{t}, \lambda_{g}, \lambda \text { - thermal conductivity of the tube material or }
\end{aligned}
$$

\section{Abbreviations}

$$
\begin{array}{ll}
\text { A } & - \text { aniline (vapour) } \\
\text { H } & - \text { hydrogen } \\
\text { MV } & - \text { Morbidelli-Varma criterion } \\
\text { NB } & - \text { nitrobenzene (vapour) } \\
\text { ODE } & - \text { ordinary differential equations } \\
\text { W } & - \text { water (vapour) }
\end{array}
$$

\section{References}

1. Stefan, D. N., Maria, G., Revista de Chimie 60 (2009) 949.

2. Maria, G., Stefan, D. N., Jl Loss Prevention in the Process Industries 23 (2010) 112. http://dx.doi.org/10.1016/j.jlp.2009.06.007

3. Maria, G., Stefan, D. N., Chemical Papers 64 (2010) 450. $\mathrm{http} / / / \mathrm{dx}$. doi.org/10.2478/s11696-010-0035-5
4. Kadam, J. V., Schlegel, M., Srinivasan, B., Bonvin, D., Marquardt, W., Journal of Process Control 17 (2007) 389. http://dx.doi.org/10.1016/j.jprocont.2006.06.006

5. Varma, A., Morbidelli, M., Wu, H., Parametric sensitivity in chemical systems, Cambridge: Cambridge University Press, 1999.

http://dx.doi.org/10.1017/CBO9780511721779

6. Li, P., Arellano-Garcia, H., Wozny, G., Computers \& Chemical Engineering 32 (2008) 25. http://dx.doi.org/10.1016/j.compchemeng.2007.05.009

7. Maria, G., Chemical process quantitative risk analysis and modelling of accident consequences. Bucharest: Printech Publ.; 2007 (in Romanian).

8. AIChE - Guidelines for chemical process quantitative risk analysis, Center for Chemical Process safety of the American Institute of Chemical Engineers, New York: AIChE, 2000.

9. Maria, G., Dinculescu, D., Khwayyir, H. H. S., Asia-Pacific J. Chem. Eng. 9 (2014) 146. http://dx.doi.org/10.1002/apj.1755

10. Maria, G., Dan. A., Asia-Pacific J. Chem. Eng. 7 (2012) 733. http://dx.doi.org/10.1002/apj.625

11. Maria, G., Dan, A., Jl. Loss Prevention in the Process Industries 25 (2012) 1033. http://dx.doi.org/10.1016/j.jlp.2012.06.007

12. Maria, G., Dan, A., Computers \& Chemical Engineering 35 (2011) 177. http://dx.doi.org/10.1016/j.compchemeng.2010.05.003

13. Maria, G., Dan, A., Revista de Chimie 62 (2011) 469.

14. Dan, A., Maria, G., Chemical Engineering \& Technology 35 (2012) 1098. http://dx.doi.org/10.1002/ceat.201100706

15. Srinivasan, B., Palanki, S., Bonvin, D., Computers \& Chemical Engineering 27 (2002) 27. http://dx.doi.org/10.1016/S0098-1354(02)00117-5

16. Hofmann, H., editor. - Ullmann's encyclopedia of industrial chemistry. Weinheim:Wiley-VCH; 2002.

17. Amon, B., Redlingshofer, H., Klemm, E., Dieterich, E., Emig, G., Chemical Engineering and Processing 38 (1999) 395. http://dx.doi.org/10.1016/S0255-2701(99)00037-9

18. Klemm, E., Amon., B., Redlingshoefer, H., Dieterich, E., Emig, G., Chemical Engineering Science 56 (2001) 1347. http://dx.doi.org/10.1016/S0009-2509(00)00357-2

19. Petrov, L., Kumbilieva, K., Kirkov, N., Applied Catalysis 59 (1990) 31 http://dx.doi.org/10.1016/S0166-9834(00)82185-5

20. Rihani, D. N., Narayanan, T. K., Doraiswamy, L. K., Ind. Eng. Chem. Process Des. Develop. 4 (1965) 403. http://dx.doi.org/10.1021/i260016a012

21. Maria, G., Ching Tao, L., Mihail, R., Revista de Chimie 34 (1983) 1093 (in Romanian).

22. Trambouze, P., Van Landeghem, H., Wauquier, J. P., Chemical reactors: Design, engineering, operation, Edition Technip, Paris, 1988.

23. Lupusor, G., Merica, E., Gorea, C., The engineering of the synthesis of aromatic intermediates, vol. 2. Technical Book Publ., Bucharest, 1981 (in Romanian).

24. Froment, G. F., Bischoff, K. B., Chemical reactor analysis and design, Wiley, New York, 1990.

25. Grewer, T., Thermal hazards of chemical reactions, Elsevier, Amsterdam, 1994.

26. Hada, S., Harrison, B. K., Jl. of Loss Prevention in the Process Industries 20 (2007) 151. http://dx.doi.org/10.1016/j.jlp.2007.02.001 
27. Quina, M. M. J., Ferreira, R. M. Q., Ind. Eng. Chem. Res. 38 (1999) 4615.

http://dx.doi.org/10.1021/ie9807295

28. Park, S. W., Himmelblau, D. M., AIChE J. 26 (1980) 168. http://dx.doi.org/10.1002/aic.690260132

29. Satterfield, C. N., Mass transfer in heterogeneous catalysis, MIT Press, Cambridge (Mass.), 1970.

30. Floarea, O., Jinescu, G., Vasilescu, P., Balaban, C., Dima, $R$., Chemical process operations and equipment, Didactica Publ., Bucharest, 1980 (in Romanian).

31. Perry R. H., Green, D. W., Maloney, J. O., Perry's Chemical Engineers' Handbook, McGraw-Hill, New York, 1997.

32. Mattson, C., Messac, A. Optimization and Engineering 6 (2005) 85.

http://dx.doi.org/10.1023/B:OPTE.0000048538.35456.45
33. Mitra, K. Chemical Engineering Science, 64 (2009) 5043. http://dx.doi.org/10.1016/j.ces.2009.08.012

34. Geletu, A. Li, P., ChemBioEng Reviews 1 (2014) 170. http://dx.doi.org/10.1002/cben.201400013

35. Vallerio, M., Telen, D., Cabianca, L., Manenti, F., Van Impe, J., Logist, F., Chemical Engineering Science 140 (2016) 201-216. http://dx.doi.org/10.1016/j.ces.2015.09.012

36. Vallerio, M., Hufkens, J., Van Impe, J., Logist, F., Expert Systems with Applications 42 (2015) 7710. http://dx.doi.org/10.1016/j.eswa.2015.05.038

37. Rao, S. S., Engineering optimization - Theory and practice, Wiley, New York, 2009. Chapter 14.10. 